University of Wollongong

Research Online

Faculty of Engineering and Information

Faculty of Engineering and Information

Sciences - Papers: Part A

Sciences

$1-1-2014$

\title{
Adaptive antenna selection for energy-efficient MIMO-OFDM wireless systems
}

Ngoc Phuc Le

University of Wollongong, pnl750@uowmail.edu.au

Le Chung Tran

University of Wollongong, Ictran@uow.edu.au

Farzad Safaei

University of Wollongong, farzad@uow.edu.au

Follow this and additional works at: https://ro.uow.edu.au/eispapers

Part of the Engineering Commons, and the Science and Technology Studies Commons

Research Online is the open access institutional repository for the University of Wollongong. For further information contact the UOW Library: research-pubs@uow.edu.au 


\title{
Adaptive antenna selection for energy-efficient MIMO-OFDM wireless systems
}

\author{
Abstract \\ In this paper, we investigate energy-efficiency in antenna selection OFDM-based systems. We show that \\ an OFDM system with conventional antenna selection approaches, including per-subcarrier selection (i.e., \\ selecting antennas independently for each subcarrier) and bulk-selection (i.e., choosing the same \\ antennas for all subcarriers), suffers from a significant loss in energy-efficiency. To achieve a better \\ energy-efficiency performance, we propose an adaptive antenna selection scheme where both the \\ number of active RF (radio frequency) chains and the antenna indices are selected depending on the \\ channel condition. This selection scheme could be implemented by exhaustive search. Moreover, we \\ develop an algorithm that achieves a near-optimal performance with much lower complexity when the \\ number of antennas is large, compared to the (optimal) exhaustive search method. Simulation results \\ demonstrate a significant improvement in terms of energy-efficiency in the proposed system compared to \\ the conventional counterparts.

\section{Keywords} \\ wireless, ofdm, mimo, systems, efficient, adaptive, energy, selection, antenna \\ Disciplines \\ Engineering | Science and Technology Studies

\section{Publication Details} \\ N. Le, L. Tran \& F. Safaei, "Adaptive Antenna Selection for Energy-Efficient MIMO-OFDM Wireless Systems," \\ in 17th International Symposium on Wireless Personal Multimedia Communications (WPMC), 2014, pp. \\ 60-64.
}




\title{
Adaptive Antenna Selection for Energy-Efficient MIMO-OFDM Wireless Systems
}

\author{
Ngoc Phuc Le, Le Chung Tran, and Farzad Safaei \\ School of Electrical, Computer and Telecommunications Engineering \\ The University of Wollongong \\ Northfields Avenue, NSW 2522, Australia \\ Emails: \{pnl750, lctran, farzad\}@uow.edu.au
}

\begin{abstract}
In this paper, we investigate energy-efficiency in antenna selection OFDM-based systems. We show that an OFDM system with conventional antenna selection approaches, including per-subcarrier selection (i.e., selecting antennas independently for each subcarrier) and bulk-selection (i.e., choosing the same antennas for all subcarriers), suffers from a significant loss in energy-efficiency. To achieve a better energy-efficiency performance, we propose an adaptive antenna selection scheme where both the number of active RF (radio frequency) chains and the antenna indices are selected depending on the channel condition. This selection scheme could be implemented by exhaustive search. Moreover, we develop an algorithm that achieves a near-optimal performance with much lower complexity when the number of antennas is large, compared to the (optimal) exhaustive search method. Simulation results demonstrate a significant improvement in terms of energyefficiency in the proposed system compared to the conventional counterparts.
\end{abstract}

Keywords- Antenna selection; energy efficiency; MIMOOFDM; UWB systems.

\section{INTRODUCTION}

Recent years have seen an increasing demand for very high data speeds in wireless multimedia applications. Also, reducing energy consumption in wireless networks has become a problem of concern among academic and industrial communities. As a result, designing high-rate wireless systems with high energy-efficiency has emerged as the main concern for future wireless networks [1]. The improvement of energyefficiency in wireless systems could be tackled at the component level, link level, or network level [1].

To date, a combination of MIMO (multi-input multi-output) techniques and OFDM (orthogonal frequency division multiplexing) has been considered as a key technique for highspeed wireless transmission [2]. This is because OFDM offers high spectral efficiency and robustness against intersymbol interference (ISI) in multipath fading channels. Meanwhile, MIMO provides an improvement in capacity and/or diversity gains [3]. In fact, MIMO-OFDM has been adopted in current and future standards, such as WiMAX, WLAN 802.11n, or LTE-Advanced, etc. Among various MIMO schemes, antenna selection appears to be a promising approach for OFDM systems. This is due to the fact that antenna selection requires a low-cost implementation and small amount of feedback information, compared to other precoding techniques [4], [5].
Many research works have considered the application of antenna selection in OFDM-based systems, e.g., in [6-9]. In general, the proposed systems can be categorized into two approaches: bulk selection (i.e., choosing the same antennas for all subcarriers) [6,9] and per-subcarrier selection (i.e., selecting antennas independently for each subcarrier) [7-9]. The main benefit of the latter over the former is that a much larger coding gain can be achieved by exploiting the frequency-selective nature of the fading channels [9]. Thus, per-subcarrier selection is very attractive for wideband communications. However, to the best of our knowledge, all these works only investigated antenna selection from either capacity or error-performance perspective. Recently, we investigated energy-efficiency in per-subcarrier antenna subset selection OFDM systems with the objective of peak-power reduction [10]. However, [10] only examined the system from a viewpoint of power-amplifier efficiency, which is not the focus of the present work. We notice that per-subcarrier selection achieves a larger coding gain than bulk-selection at a cost of additional power consumption due to multiple active RF (radio frequency) chains. Thus, some important issues arise when investigating antenna selection from an energyefficiency viewpoint, which has not been addressed in the literature. In particular, it is not clear if the two conventional selection approaches are optimal in terms of energyefficiency. If not, then selecting antennas in OFDM systems for improved energy-efficiency remains an open question.

In this paper, we investigate energy-efficiency in antenna selection MIMO-OFDM systems. The main contributions of this work are as follows:

- The efficacy of MIMO-OFDM systems with different conventional antenna selection schemes is evaluated from an energy-efficiency perspective. It is shown that these systems are not effective with respect to energy-efficiency.

- An adaptive antenna selection approach is proposed to improve the energy-efficiency in MIMO-OFDM systems. Specifically, both the number of active RF chains and the antennas are selected to maximize the energy-efficiency.

- An algorithm to implement the proposed adaptive selection scheme that could attain a near-optimal energy-efficiency with low-complexity is developed.

The remainder of the paper is organized as follows. In Section II, an energy-efficient OFDM system with the conventional antenna selection is described. In Section III, we 


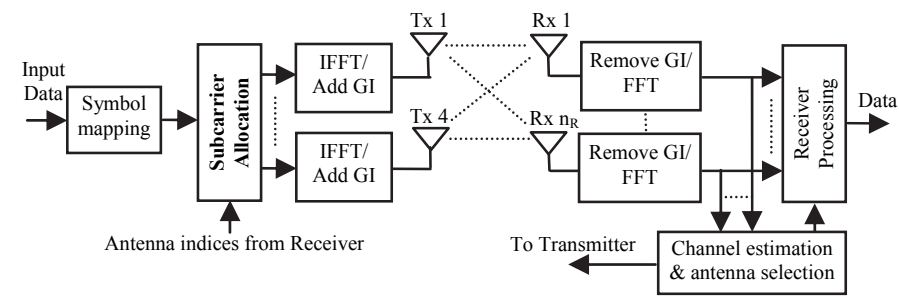

Fig.1. Block diagram of an antenna selection MIMO-OFDM system.

propose an adaptive selection scheme with a low-complexity algorithm. In Section IV, we provide simulation results and perform in-depth evaluations of energy-efficiency in the considered systems. Finally, Section V concludes the paper.

\section{ENERGY-EFFICIENCY BASED ANTENNA SELECTION FOR OFDM WIRELESS SYSTEMS}

\section{A. System Model}

We consider an OFDM system with $K$ subcarriers, $n_{T}$ transmit antennas, and $n_{R}$ receive antennas as shown in Fig.1. At the transmitter, the input data stream is mapped onto $M$ QAM ( $M$-ary Quadrature Amplitude Modulation) constellations. For the $k^{\text {th }}$ subcarrier, we denote $u(k)$ and $x_{i}(k), 1 \leq i \leq n_{T}, 0 \leq k \leq K-1$, to be the symbols that the allocation block takes at its input and outputs at its $i^{\text {th }}$ output, respectively. This block assigns the data symbol $u(k)$ to the selected antenna at the $k^{\text {th }}$ subcarrier based on a selection criterion. As a result, only one element in a vector $\mathbf{x}(k)=\left[x_{1}(k), x_{2}(k), \ldots, x_{n_{T}}(k)\right]^{T}$ contains the data symbol $u(k)$, whereas the others are zeros. The output sequences from the subcarrier allocation block are then fed into $K$-point IFFT blocks. Each time-domain OFDM signal is then added with a guard interval (GI) before being transmitted via its corresponding transmit antenna. Note that the transmit branch corresponding to the output of the allocation block that is not allocated any data symbol is turned off to save energy ${ }^{1}$.

At the receiver, the received signal at each antenna is fed into the FFT block after the GI is removed. The system model in the frequency domain corresponding to the $k^{\text {th }}$ subcarrier can be expressed as [2]

$$
\mathbf{y}(k)=\mathbf{H}(k) \mathbf{x}(k)+\mathbf{n}(k)=\mathbf{h}(k) u(k)+\mathbf{n}(k),
$$

where a $n_{R} \times n_{T}$ matrix $\mathbf{H}(k)$ denotes the subchannel matrix associated with the $k^{\text {th }}$ subcarrier, and $\mathbf{h}(k)$ indicates the effective channel vector obtained by selecting the column of $\mathbf{H}(k)$ that is corresponding to the chosen transmit antenna on the $k^{\text {th }}$ subcarrier. Also, $\mathbf{y}(k)=\left[y_{1}(k), y_{2}(k), \ldots, y_{n_{R}}(k)\right]^{T}$, and $\mathbf{n}(k)=\left[n_{1}(k), n_{2}(k), \ldots, n_{n_{R}}(k)\right]^{T}$, where $y_{j}(k)$ and $n_{j}(k)$ denote the received signal and the noise at the $j^{\text {th }}$ receive antenna, respectively. Here, the noise is modeled as a Gaussian random variable with zero mean and variance $\sigma_{n}^{2}$. Assume that the receiver uses a MRC (maximum ratio

${ }^{1}$ This system model is used for all the considered selection methods in this paper for convenience. In reality, bulk selection needs only one RF chain and performs RF switching rather than subcarrier allocation.

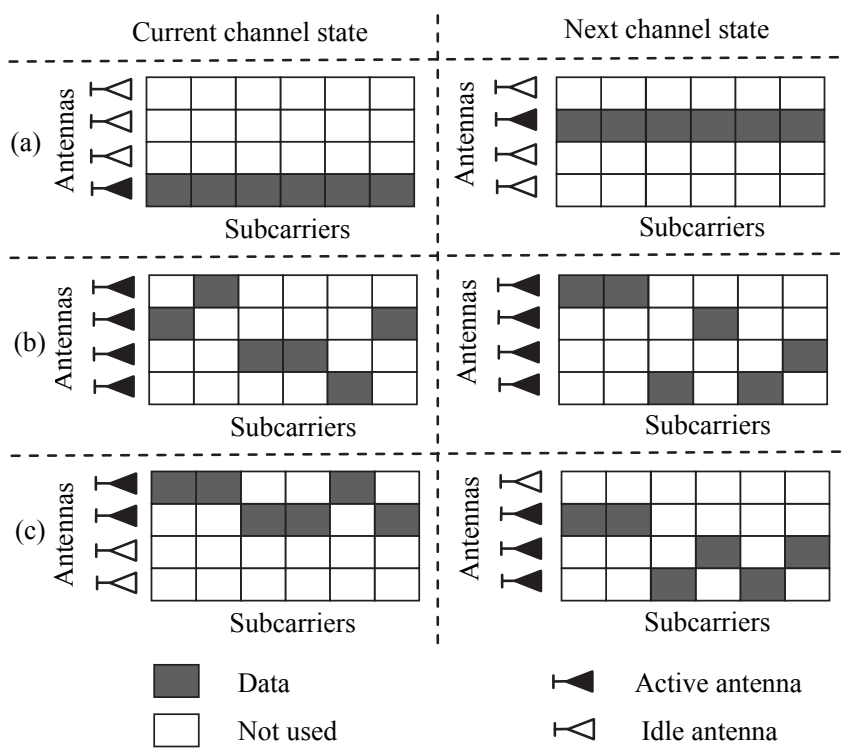

Fig. 2. Illustration of antenna selection methods: (a) Bulk selection; (b) Per -subcarrier selection; (c) Proposed adaptive selection; $\left(n_{T}=4\right.$ and $K=6$ ).

combining) for signal detection, the detected signal is given as

$$
z(k)=\|\mathbf{h}(k)\|^{2} u(k)+\mathbf{h}^{H}(k) \mathbf{n}(k),
$$

where $(.)^{H}$ and $\|.\|^{2}$ denote Hermitian transpose and a squared norm of a vector, respectively. In this work, for simplicity, we assume that power loading across subcarrier is not an option (i.e., equal power allocation). This assumption may be required in some systems, e.g., UWB (ultra-wideband) systems, due to the regulation of a power spectral mask.

\section{B. Conventional Antenna Selection}

In this subsection, we investigate the conventional selection approaches (i.e., bulk selection and per-subcarrier selection) from an energy-efficiency viewpoint. To quantify the fundamental limits of the system, we consider an energyefficiency (bits/Joule) defined as a ratio between the achievable rate and the total power consumption, i.e., $[10,11]$

$$
E E=C / P_{\text {total }},
$$

where $C$ denotes the achievable rate (capacity) and $P_{\text {total }}$ is the power consumption, i.e., $P_{\text {total }}=K P_{t} / \eta+n_{R F} P_{R F}+P_{s p}$, where $\eta$ is the power efficiency of high-power amplifier (HPA), $n_{R F}$ is the number of active RF chains, $P_{t}$ is the actual transmitted power per subcarrier, $P_{R F}$ is the RF front-end power consumption in each transmit branch excluding the associated HPA, and $P_{s p}$ is the baseband processing power consumption. From an energyefficiency perspective, antenna selection schemes aim to select antennas to maximize the $E E$ value in (3).

\section{1) Bulk antenna selection}

In the bulk selection, only one among $n_{T}$ available antennas is used to transmit data (see Fig.2a for illustration). Thus, this method requires only one active RF chain, i.e., $n_{R F}=1$. Given the fixed transmitted power $P_{t}$, it can be seen from (3) that the total power $P_{\text {total }}$ is fixed. Therefore, to maximize $E E$ in (3), we only need to select the antenna that maximizes the achievable rate $C$ (bits/s). Let $I_{i}(k)$ denote the mutual 
information (MI) (bits/s/Hz) associated with the $k^{\text {th }}$ subcarrier and the $i^{\text {th }}$ transmit antenna. Then, we have [3]

$$
I_{i}(k)=\log _{2}\left(1+\rho\left\|\mathbf{h}_{i}(k)\right\|^{2}\right),
$$

where $\rho$ is the SNR (signal-to-noise ratio) value. The accumulated MI across subcarriers associated with the $i^{\text {th }}$ antenna can be expressed as

$$
\bar{I}_{i}=\sum_{k=0}^{K-1} I_{i}(k) .
$$

Note that the achievable rate (i.e., ergodic capacity per OFDM symbol) is given as $C=(B / K) \times \mathrm{E}_{\mathbf{H}}\left\{\bar{I}_{i}\right\}$, where $\mathrm{E}_{\mathbf{H}}\{\cdot\}$ denotes an expectation operation over the fading channel distribution, and $B$ is the system bandwidth. The optimal antenna that maximizes $E E$ in (3) is determined by

$$
\hat{i}=\arg \max _{i=1, \ldots, n_{T}} \bar{I}_{i} \text {. }
$$

\section{2) Per-subcarrier antenna selection}

Unlike the bulk selection, in per-subcarrier selection, antennas are selected independently for each subcarrier (see Fig. $2 \mathrm{~b}$ for illustration). Thus, all equipped antennas are active, i.e., $n_{R F}=n_{T}$. It is straightforward to show that the $E E$ value in (3) is maximized when antennas are selected to maximize $I_{i}(k), \forall k=1,2, \ldots, K$. In other words, the optimal antenna associated with the $k^{\text {th }}$ subcarrier is selected as

$$
\hat{i}(k)=\arg \max _{i=1, \ldots, n_{T}} I_{i}(k) \text {. }
$$

\section{Proposed Adaptive Antenna Selection Scheme FOR IMPROVED ENERGY-EFFICIENCY}

In Section II, we have considered the MIMO-OFDM system with the conventional antenna selection approaches. It is noted that the number of active RF chains in the bulk selection and per-subcarrier selection are $n_{R F}=1$ and $n_{R F}=n_{T}$, respectively. However, it is not guaranteed that the system operating with such a fixed value of $n_{R F}$ is optimal from an energy-efficiency perspective. The reason is that the $E E$ value defined in (3) depends on many factors, including the channel condition, the actual transmit power, as well as the power consumed by the electronics circuits (mainly $n_{R F} \mathrm{RF}$ chains). When one antenna (i.e., one RF chain) is activated/deactivated, the system will achieve a higher/lower capacity (cf.(5)-(7)). Meanwhile, the power consumption due to RF chains is increased/decreased. Consequently, whether the $E E$ value is increased or not depends on the changes in the capacity and consumed power. Given a fixed value of $P_{R F}$, to achieve an improved $E E$, whether an antenna should be activated or deactivated depends on the channel condition. Based on these observations, we propose to improve $E E$ by adaptively selecting both the number of active RF chains and the transmit antenna indices (see Fig. 2c). This proposed selection approach can be implemented by either an exhaustive search or a lowcomplexity algorithm, which are described in detail below.

\section{A. Exhaustive Search Method}

When the number of transmit antennas $n_{T}$ is small, an exhaustive search method can be used to achieve an optimal antenna allocation. In particular, this method checks all possible sets of antennas, and selects the set that attains the
TABle I. A Proposed Low-Complexity Selection Algorithm.

(1): Initial setting: A set of unallocated subcarriers $\Omega_{0}=\{1,2, \ldots, K\}$, A set of available transmit antennas $S_{0}=\left\{1,2, \ldots, n_{T}\right\}$, A set of selected antennas $P_{0}=\{\varnothing\}$, and $E E_{0}=0$.

(2): Calculate the instantaneous mutual information $I_{i}(k), \forall i \in S_{0}, \forall k \in \Omega_{0}$, using Eq. (4).

(3): for $m=1: n_{T}$ do

(4): Calculate the accumulated MI across unallocated subcarriers (used to select antenna)

$$
\Psi_{i}^{(m)}=\sum_{k \in \Omega_{m-1}} I_{i}(k), i \in S_{m-1} .
$$

(5): Select the $\hat{i}_{m}$ antenna that satisfy $\hat{i}_{m}=\underset{i \in S_{m-1}}{\arg \max _{i}^{(m)}}$.

(6): Add $\hat{i}_{m}$ to the set of selected antennas, i.e., $P_{m}=\left\{P_{m-1}, \hat{i}_{m}\right\}$.

(7): Assign the selected antenna $\hat{i}_{m}$ to the subcarriers $l$ that satisfy

$$
I_{\hat{i}_{m}}(l)=\arg \max _{i \in S_{m-1}} I_{i}(l) .
$$

(8): Update the set of unallocated subcarriers as $\Omega_{m}=\Omega_{m-1}-\bar{\Omega}$, where $\bar{\Omega}$ is the set of the allocated subcarriers in Step (7).

(9): Select antennas in $P_{m}$ for the remain unallocated subcarriers via

$$
\hat{i}_{m}(k)=\underset{i \in P_{m}}{\arg \max _{i}} I_{i}(k), \forall k \in \Omega_{m} .
$$

(10): Calculate the accumulated MI corresponding to the set $P_{m}$ (used to calculate $E E$ )

$$
\bar{I}_{m}=\sum_{k=0}^{K-1} I_{\hat{i}_{m}(k)}(k)
$$

(11) Calculate $E E$ using Eq.(3): $E E_{m}=(B / K) \times \bar{I}_{m} /\left(K P_{t} / \eta+m P_{R F}+P_{s p}\right)$.

(12): if $E E_{m}>E E_{m-1}$

(13): $\quad P_{\text {select }} \leftarrow P_{m}$,

(14): $\quad \hat{i}_{\text {select }}(k) \leftarrow \hat{i}_{m}(k), k=1,2, \ldots, K$.

(15): end if

(16): Update the available antenna index set as $S_{m}=S_{0}-P_{m}$.

(17): end for

(18): The set of selected antennas and the allocation pattern for a maximum $E E$ are $P_{\text {select }}$ and $\hat{i}_{\text {select }}(k), k=1,2, \ldots, K$, respectively.

highest $E E$. Note that the number of possible sets is $\mathrm{C}_{1}^{n_{T}}+\mathrm{C}_{2}^{n_{T}}+\ldots+\mathrm{C}_{n_{T}}^{n_{T}}=2^{n_{T}}-1$ (where $\mathrm{C}_{m}^{n}$ denotes the binomial coefficient), which incurs very high complexity if $n_{T}$ is large. Thus, a lower complexity method is preferred.

\section{B. Proposed Antenna Selection Algorithm}

To realize the proposed adaptive selection scheme with low complexity, we develop a selection algorithm described in Table I. This algorithm selects antennas in an incremental fashion and is based on the following principles:

A1: Given a fixed number of active antennas, a larger capacity leads to a larger $E E$ value (cf.(3)). Thus, if we already selected the set $P_{m}$ consisting of $m$ antennas, the optimal antenna $\hat{i}_{m+1}$ that is added to create the set $P_{m+1}$ is the antenna that makes $P_{m+1}$ achieve the largest accumulated MI (mutual information). A2: If the MI at the $l^{\text {th }}$ subcarrier on the antenna $\hat{i}_{m}, \hat{i}_{m} \in P_{m}$ satisfies $I_{\hat{i}_{m}}(l)=\underset{i \in S_{m-1}}{\operatorname{argmax}} I_{i}(l)$, where $S_{m-1}=\left\{1,2, \ldots, n_{T}\right\}-P_{m-1}$, then the antenna $\hat{i}_{m}$ is immediately selected for the $l^{\text {th }}$ subcarrier 
TABle II. Simulation Parameters.

\begin{tabular}{|l|l|}
\hline \multicolumn{1}{|c|}{ Parameter } & \multicolumn{1}{|c|}{ Value } \\
\hline Bandwidth & $B=528 \mathrm{MHz}$ \\
Modulation & 4-QAM \\
FFT size & $K=128$ \\
Circuit powers & $P_{R F}=200 \mathrm{~mW} ; P_{s p}=100 \mathrm{~mW}$ \\
Path loss & $81 \mathrm{~dB}$ \\
Noise power & $-84 \mathrm{dBm}$ \\
Power amplifier efficiency & $35 \%$ \\
IEEE 802.15.3a channel model & $\mathrm{CM} 1$ \\
Number of channel realizations & $10^{3}$ \\
\hline
\end{tabular}

when it is added to the set $P_{m-1}$. Note that $I_{\hat{i}_{m}}(l)$, the largest MI value on the allocated $l^{\text {th }}$ subcarrier, will be used as the MI on the $t^{\text {th }}$ subcarrier when measuring the accumulated MI for all $n_{T}$ sets $P_{m}, m=1,2, \ldots, n_{T}$. Thus, the MI corresponding to the $l^{\text {th }}$ subcarrier on the remaining available antennas will not be considered when evaluating the accumulated MI. Consequently, the optimal antenna $\hat{i}_{m+1}$ mentioned in $\mathbf{A} \mathbf{1}$ is the one that has the largest accumulated MI measured only over the set of its unallocated subcarriers.

\section{Complexity Comparison}

With respect to a comparison of complexity between the proposed algorithm and the exhaustive search, we consider the number of allocation operations as a metric. In the exhaustive search, there are $\left(2^{n_{T}}-1\right)$ possible sets, and each set needs $K$ allocations for $K$ subcarriers. Thus, the number of allocations is $\zeta_{\text {opt }}=K\left(2^{n_{T}}-1\right)$. Meanwhile, the $m^{\text {th }}\left(m=1,2, \ldots, n_{T}\right)$ loop in the proposed algorithm searches for $\left(n_{T}-m+1\right)$ sets and performs $\left|\Omega_{m-1}\right|$ allocations for each set. Here, $\left|\Omega_{m-1}\right|$ denotes the cardinality of the set $\Omega_{m-1}$. Thus, the proposed algorithm requires only $\zeta_{\text {sub }}=\sum_{m=1}^{n_{T}}\left(n_{T}-m+1\right)\left|\Omega_{m-1}\right|$ allocations. In the worst case, i.e., $\left|\Omega_{m-1}\right|=K, \forall m=1, \ldots, n_{T}$, we have $\zeta_{\text {sub }}=\sum_{m=1}^{n_{T}}\left(n_{T}-m+1\right) K=K n_{T}\left(n_{T}+1\right) / 2$, which is still much smaller than $\zeta_{\text {opt }}$ when $n_{T}$ is large.

\section{Simulation Results And Discusstions}

In this section, we provide some simulation results to evaluate the energy-efficiency in antenna selection OFDM systems as well as demonstrate the effectiveness of the proposed selection scheme. The legacy WiMedia MB-OFDM UWB (Multiband-OFDM UWB) standard [12] is adopted in our simulations. The simulation parameters are listed in Table II. The channel model of CM1, defined in the IEEE 802.15.3a channel model [13], is based on a measurement of a line-ofsight scenario where the transmitter-receiver distance is up to $4 \mathrm{~m}$. Moreover, the multipath gains are modeled as independent log-normally distributed random variables.

\section{A. Efficacy of the Proposed Antenna Selection Scheme}

We first evaluate the effectiveness of the different antenna selection methods from an energy-efficiency perspective. Fig. 3 plots the energy-efficiency versus the power $P_{t}$ in the

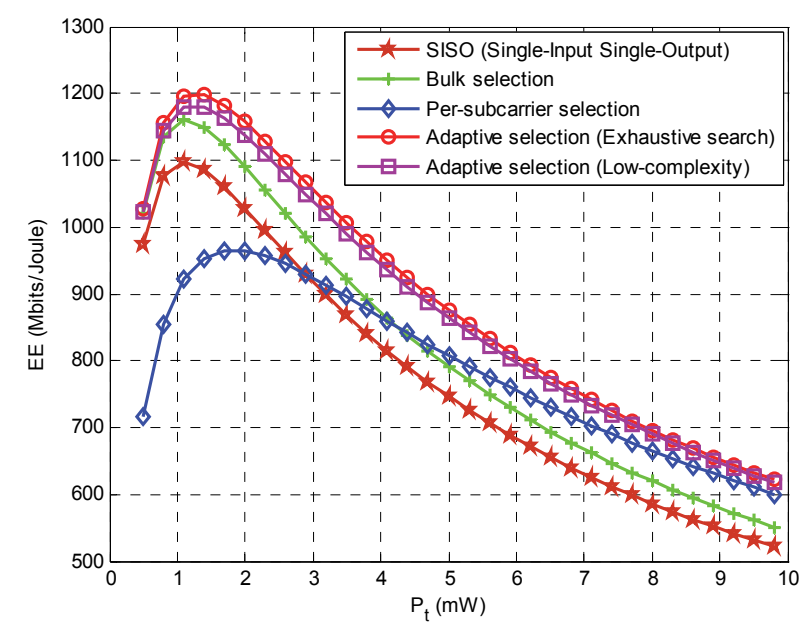

Fig. 3. Comparison of the energy-efficiency in the systems $\left(n_{T}=4, n_{R}=1\right)$.

considered systems. The obtained results demonstrate the following. First, the system with the conventional selection methods exhibits lower energy-efficiency. In particular, the bulk-selection scheme is only effective when $P_{t}$ is small Meanwhile, the per-subcarrier antenna selection is only effective in the large $P_{\mathrm{t}}$ region. The former suffers from a loss in energy-efficiency in the high $P_{\mathrm{t}}$ region due to the fact that, in this region, $K P_{\mathrm{t}} / \eta$ dominates $\left(P_{\mathrm{RF}}+P_{\mathrm{sp}}\right)$. Thus, activating multiple RF chains will achieve a larger capacity, while incurring a very small additional power consumption due to RF chains (cf.(3)). In other words, in this region, multiple antennas (rather than only one antenna) should be activated to achieve an improved $E E$. The behavior of the latter can also be explained in a similar manner. Second, the proposed antenna selection method achieves a better $E E$ performance than the conventional counterparts. The reason is that the proposed method can adapt the number of active RF chains according to the channel condition. As a result, in the small and large $P_{\mathrm{t}}$ regions, it could offer a similar energy-efficiency as the bulkselection and per-subcarrier selection, respectively. In the remaining region, it outperforms the two conventional methods as the optimal number of active RF chains $n_{R F}$ for the maximum energy-efficiency is $1<n_{R F}<n_{T}$. Recall that the number of active RF chains in the conventional bulk-selection and the per-subcarrier selection methods are always one and $n_{T}$, respectively. Hence, they cannot achieve the maximum $E E$. Third, the energy-efficiency achieved with the proposed lowcomplexity algorithm is relatively close to that with the exhaustive search method, which demonstrates the effectiveness of this algorithm from a practical viewpoint.

\section{B. Energy-Efficiency versus the Number of Antennas}

In the literature, where antenna selection OFDM systems are investigated from capacity or error performance perspective, it is shown that the more antennas are equipped, the larger capacity or the better error-rate is achieved (see, e.g. [4]). When the energy-efficiency metric is considered in our work, the relation between $E E$ and the number of equipped transmit antennas is shown in Fig. 4. It can be seen that, in the bulk selection and proposed adaptive selection systems, the $E E$ 


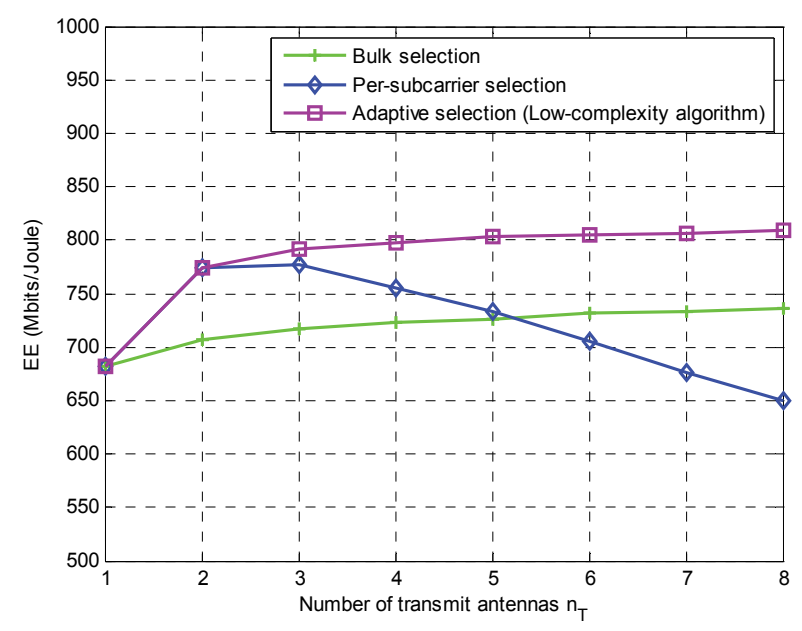

Fig. 4. Energy-efficiency versus the number of transmit antennas $\left(P_{t}=6 \mathrm{~mW}, n_{R}=1\right)$

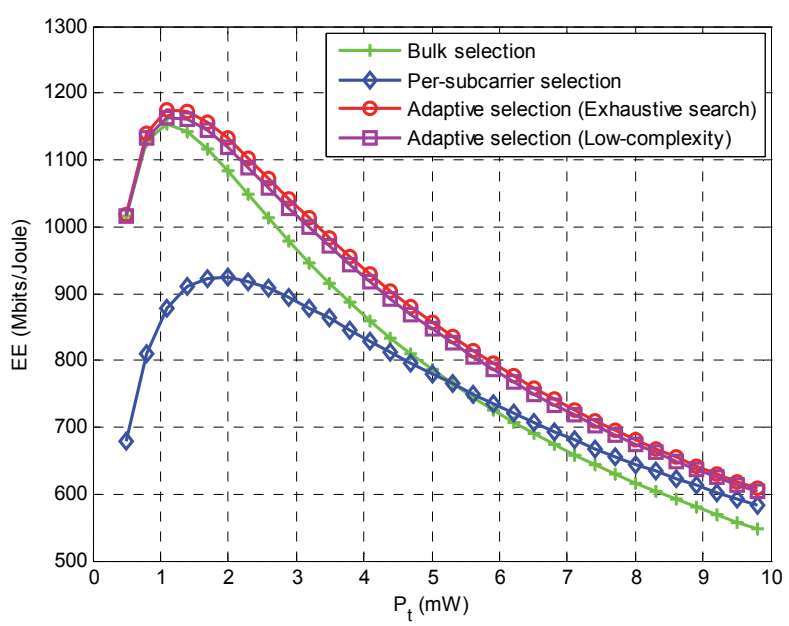

Fig. 5. Comparison of energy-efficiency under spatially correlated channels (correlation coefficient of $0.7, n_{T}=4, n_{R}=1$ ).

values increase when the number of antennas $n_{T}$ is increased. However, these $E E$ values become saturated when $n_{T}$ is very large. Meanwhile, in the per-subcarrier selection system, the $E E$ value first increases and then decreases. This behavior can be explained by the fact that, when $n_{T}$ becomes large, the increased power consumption due to the associated RF chains has more impact on $E E$ than the capacity improvement does, which reduces the $E E$ value (cf.(3)). It is also important to note that the proposed system outperforms its counterparts. In addition, the improvement in $E E$ depends on a relative comparison between the transmitted power and the power consumed by the electronics circuits.

\section{Impact of Spatial Correlation on Energy-Efficiency}

We finally consider the effect of spatial correlation on the energy-efficiency in the antenna selection OFDM systems. The spatially correlated channel is modeled as $\mathbf{H}=\mathbf{H}_{i i d} \mathbf{R}_{T}^{1 / 2}$ [14], where $\mathbf{R}_{T}$ is the $n_{T} \times n_{T}$ transmit correlation matrix. Also, $\mathbf{H}_{i i d}$ denotes the $n_{R} \times n_{T}$ channel matrix consisting of independent channel realizations. It can be seen from Fig. 5 and Fig. 3 that the presence of spatial correlation reduces the energy-efficiency. This makes sense as, given a fixed number of antennas, the correlation between transmit antennas reduces the system capacity [14], which in turn lowers the energyefficiency (cf.(3)). However, it is important to note that the proposed system remains superior with respect to energyefficiency compared to the conventional counterparts.

\section{CONCLUSIONS}

This paper has investigated the energy-efficiency in MIMO-OFDM systems employing different antenna selection schemes. Several important factors that affect the energyefficiency have been evaluated, including the relation between actual transmitted power and the power consumed by the transceiver circuits, the number of equipped antennas, and the correlation between antennas. It has been shown that the conventional antenna selection methods exhibit a loss of energy-efficiency. Thus, the adaptive antenna selection scheme has been proposed to deal with this issue. Moreover, the low-complexity algorithm to realize this adaptive selection scheme has been developed. Simulation results show that the proposed selection scheme with this algorithm outperforms (in terms of energy-efficiency) the conventional counterparts.

\section{REFERENCES}

[1] Z. Hasan, H. Boostanimehr, V. K. Bhargava, "Green cellular networks: A survey, some research issues and challenges," IEEE Commun. Surveys \& Tutorials, vol. 13, no. 4, pp. 524-540, Nov. 2011.

[2] G. L. Stuber, J. R. Barry, S. W. Mclaughlin, Y. Li, M. A. Ingram, and T. G. Pratt, "Broadband MIMO-OFDM wireless communications," Proc. IEEE, vol. 92, no. 2, pp. 271-294, Feb. 2004.

[3] D. Tse and P. Viswanath, Fundamentals of Wireless Communication, Cambridge University Press, USA, 2005.

[4] A. F. Molisch and M. Z. Win, "MIMO systems with antenna selection," IEEE Microwave Magazine, vol. 5, no. 1, pp. 45-56, March 2004.

[5] C. M. Vithanage, J. P. Coon, and S. C. J. Parker, "On capacity-optimal precoding for multiple antenna systems subject to EIRP restrictions," IEEE Trans. Wireless Commun., vol. 7, no. 12, pp. 5182-87, Dec. 2008.

[6] H. Zhang, A. F. Molisch, and J. Zhang, "Appling antenna selection in WLAN for achieving broadband multimedia communications," IEEE Transactions on Broadcasting, vol. 52, no. 4, pp. 475-482, Dec. 2006.

[7] M. Sandell and J. Coon, "Per-subcarrier antenna selection with power constraints in OFDM systems," IEEE Transactions on Wireless Communications, vol. 8, no. 2, pp. 673-677, Feb. 2009.

[8] N. P. Le, F. Safaei, and L. C. Tran, “Transmit antenna subset selection for high-rate MIMO-OFDM systems in the presence of nonlinear power amplifiers," EURASIP Journal on Wireless Communications and Networking 2014. doi:10.1186/1687-1499-2014-27.

[9] H. Zhang and R. U. Nabar, "Transmit antenna selection in MIMOOFDM systems: bulk versus per-tone selection," in Proc. IEEE Intern. Conf. Commun., ICC 2008, pp. 4371-4375, May 2008.

[10] N. P. Le, L. C. Tran, and F. Safaei, "Energy-efficiency analysis of persubcarrier antenna selection with peak-power reduction in MIMOOFDM wireless systems," International Journal of Antennas and Propagation 2014. doi:10.1155/2014/313195.

[11] S. Cui, A. Goldsmith, and A. Bahai, "Energy-constrained modulation optimization," IEEE Transactions on Wireless Communications, vol. 4 , no. 5, pp. 2349-2360, Sept. 2005.

[12] A. Batra et al., "Multiband OFDM physical layer specification," WiMedia Alliance, Release 1.5, August 2009.

[13] J. Foerster et al., "Channel modeling sub-committee report final," IEEE P802.15-02/490r1-SG3a, Feb. 2003.

[14] T. Kaiser, F. Zheng, and E. Dimitrov, "An overview of ultrawideband systems with MIMO," Proc. IEEE, vol. 97, no. 2, Feb. 2009. 